\title{
Conductivity Degradation Study of PEDOT: PSS Films under Heat Treatment in Helium and Atmospheric Air
}

\author{
Evangelos Vitoratos ${ }^{1}$, Sotirios Sakkopoulos ${ }^{1}$, Nikolaos Paliatsas ${ }^{1}$, Konstantinos Emmanouil ${ }^{1}$, \\ Stelios A. Choulis ${ }^{2}$ \\ ${ }^{1}$ Department of Physics, University of Patras, Patras, Greece \\ ${ }^{2}$ Molecular Electronics and Photonics Research Unit, Department of Mechanical Engineering and \\ Materials Science and Engineering, Cyprus University of Technology, Limassol, Cyprus \\ Email: sakkop@physics.upatras.gr
}

Received December 4, 2011; revised January 9, 2012, accepted January 15, 2012

\begin{abstract}
PEDOT:PSS buffer layers have been processed with the standard annealing step used for organic solar cells device applications. The d.c. conductivity $\sigma$ as a function of temperature for two heating rates under He and atmospheric air was studied. Moreover, the stability of the conductivity was investigated at different temperatures and environments vs time. The main results can be summarized in the following: the increase of $\sigma$ due to the thermal activation of the carriers and the improvement of the crystallinity compete with the decrease of $\sigma$ resulting from the irreversible structural degradation of the polymer chains promoted by the oxygen and moisture of the atmospheric air. The heat treatment time and the temperature at which the two competing mechanisms result in a maximum of the electrical conductivity have been determined and results are discussed relevant to organic optoelectronic devices containing PEDOT: PSS buffer layers.
\end{abstract}

Keywords: Conducting Polymers; Buffer Layers Degradation; PEDOT:PSS; Thermal Treatment; Organic Solar Cells; Organic Electronics

\section{Introduction}

The homogeneous polymer complex of poly-ethylenedioxythiophene (PEDOT), oxidized with polystyrene sulphonate (PSS), has extensively been used as buffer layer in organic optoelectronic applications due to its high electrical conductivity and transparency in the visible region [1-5]. However, degradation related issues of PEDOT: PSS can be a parameter that might influence the lifetime performance of organic optoelectronic devices and other applications in which it participates [6-11]. High temperature storage in the dark is one of the accelerated lifetime test performed for determining degradation parameters for organic solar cell device applications. Thus, a better understanding of the mechanisms affecting conductivity, especially under atmospheric air containing oxygen and moisture within the temperature range in which a device is expected to operate, is critical for the continuing progress of organic opto-electronics.

Further understanding of the parameters which influence the conductivity of PEDOT:PSS can help us to identify methods to improve the electrical properties and thermal stability of this material. Degradation is connected to irreversible morphological and chemical changes, which continue to be investigated $[6,12,13]$, despite the fact that pristine PEDOT and PEDOT:PSS have been widely us- ed in the past. It is well known that pristine PEDOT films are stable under heat treatment and decomposition occurs above $663 \mathrm{~K}$ [14]. Moreover, X-ray investigation reveals that heating at $473 \mathrm{~K}$ increases crystallinity in PEDOT [15]. The stability of the transparent PEDOT:PSS polymer complex at ambient atmosphere conditions is crucial for the performance of organic electronic devices and it is still under investigation.

In this paper PEDOT:PSS buffer layers have been processed with the standard annealing step used for organic solar cells device applications. We report the results of the post-processing thermal treatment on the electrical conductivity of PEDOT:PSS films for different heating rates, temperatures and time intervals, under inert $\mathrm{He}$ and atmospheric air. The purpose of this study is to identify the detailed competition mechanisms which contribute to the change of the electrical conductivity and comprehend the underlying physical processes. The conditions under which a maximum of the electrical conductivity is obtained can be relevant to the lifetime of organic electronic devices under high temperature storage.

\section{Experimental}

All measurements were performed on $120 \mathrm{~nm}$ thick PEDOT: PSS films spin coated on pristine polyethylene te- 
rephthalate (PET) substrates. An aqueous dispersion of PEDOT: PSS (CLEVIOS PH 500, H. C. Starck, GmbH, Germany) was used, where the ratio PEDOT to PSS was $1: 2.5$ by weight.

D.C. conductivity measurements were performed using a four probe method [16]. The temperature was monitored by an Oxford intelligent temperature controller (ITC4). A Keithley 2400 sourcemeter and a Keithley 182 sensitive digital voltmeter were used to control the current and voltage respectively and parameters were measured automatically via a PC.

The influence of the thermal expansion of the PET substrate was also investigated. Over the entire temperature range $(80 \mathrm{~K}-450 \mathrm{~K})$ the change of $\sigma$ and $\sigma / \sigma_{0}$ due to PET expansion [17] was calculated to be about $0.5 \%$. These error bars are so small that they are hidden into the size of the experimental points.

Moreover, the calculation of the accidental experimental errors gave the values $\delta \sigma= \pm 0.01 \mathrm{~S} / \mathrm{cm}$ and $\delta\left(\sigma / \sigma_{0}\right)=$ \pm 0.003 .

\section{Results and Discussion}

\subsection{Influence of the Heating Rate on the Electrical Conductivity of PEDOT:PSS under $\mathrm{He}$ and Atmospheric Air}

In Figure 1 the reduced conductivity $\sigma / \sigma_{0}$, where $\sigma_{0}$ is the conductivity at $80 \mathrm{~K}$, is shown as a function of temperature $\mathrm{T}$ for two different heating rates, 5 and $15 \mathrm{~K} / \mathrm{min}$, under $\mathrm{He}$ and atmospheric air.

These heating rates have been chosen after preliminary measurements, leading to the conclusion that $15 \mathrm{~K} / \mathrm{min}$ is the maximum heating rate that ensured thermal equilibrium of the samples at each temperature. The $5 \mathrm{~K} / \mathrm{min}$ rate was sufficiently slow, so all measurements were taken in a state of thermal equilibrium [18].

As it is shown in Figure 1, the reduced conductivity values obtained under air are lower than those obtained under He. This can be attributed to irreversible structural modifications arising in the PEDOT chains and the counter ions by oxygen and moisture [12-14]. Moreover, the slower rate of heating creates higher conductivity values than the quicker one. This indicates that with slower heating rate $(5 \mathrm{~K} / \mathrm{min})$, the morphological changes which lead to the improvement of the crystallinity have enough time to be better developed than during the quicker heating rate $(15 \mathrm{~K} / \mathrm{min})$. X-ray data have revealed that heating PEDOT: PSS increases its crystalline order [15,19].

The electrical conductivity $\sigma$ increases with temperature $\mathrm{T}$ up to about $400 \mathrm{~K}$ and then decreases for higher temperatures. PEDOT:PSS has a heterogeneous structure, in which small conductive grains $20-50 \mathrm{~nm}$ in diameter, are separated by regions rich in insulating PSS [20,21]. In the conductivity mechanisms of such a heterogeneous material the carrier conduction into the grains and the tunneling of the charge carriers between them are contributing [22]. As it can be seen in Figure 1, the conductivity exhibits a maximum at a certain temperature $T_{m}$ from both sides of which the slope $\mathrm{d} \sigma / \mathrm{dT}$ has opposite sign. This is an indication that a "non-metallic" $(\mathrm{d} \sigma / \mathrm{dT}>0)$ thermally activated conductivity prevails for $\mathrm{T}<\mathrm{T}_{\mathrm{m}}$. Four mechanisms determine the overall electrical conductivity: Two of them, the heat activated tunneling of carriers between the conductive grains and the improvement of the crystallinity into them, increase conductivity. The other two mechanisms, the interposition of oxygen and moisture and the interruption of the chains because of the heating, decrease conductivity. For $\mathrm{T}>\mathrm{T}_{\mathrm{m}}$, the two latter mechanisms prevail giving a "metallic" $(\mathrm{d} \sigma / \mathrm{dT}<0)$ transport. This behavior has been observed in many conductive polymers with a heterogeneous structure similar to that of PEDOT: PSS [23,24].

Another remarkable feature of the heat treatment can be deduced from Figure 1. The characteristic temperature $\mathrm{T}_{\mathrm{m}}$ depends exclusively on the heating rate, taking the values $(384 \pm 4) \mathrm{K}$ and $(405 \pm 2) \mathrm{K}$ for the heating rates of $5 \mathrm{~K} / \mathrm{min}$ and $15 \mathrm{~K} / \mathrm{min}$ respectively, independent from the surrounding atmosphere $\mathrm{He}$ or air. This may be attributed to the following reasons: as the temperature rises, the activation of the carriers and the improvement of the crystalline order increase the electrical conductivity of the polymer, but at a certain temperature $\mathrm{T}_{\mathrm{m}}$ the simultaneous structural degradation of the PEDOT:PSS chains prevails, resulting in the overall decrease of the conductivity for higher temperatures. The fact that $T_{m}$ depends only on the heating rate and not on the surrounding atmosphere, indicates that the damage in the polymer chains caused by the temperature rise is independent from the corresponding degradation imposed to them by oxygen and moisture. For the heating rate of 5 $\mathrm{K} / \mathrm{min}_{\mathrm{m}}$ appears about 20 degrees lower than that for $15 \mathrm{~K} / \mathrm{min}$, indicating that at the slower heating, the damage due to the temperature rise is greater, than in the faster heating rate.

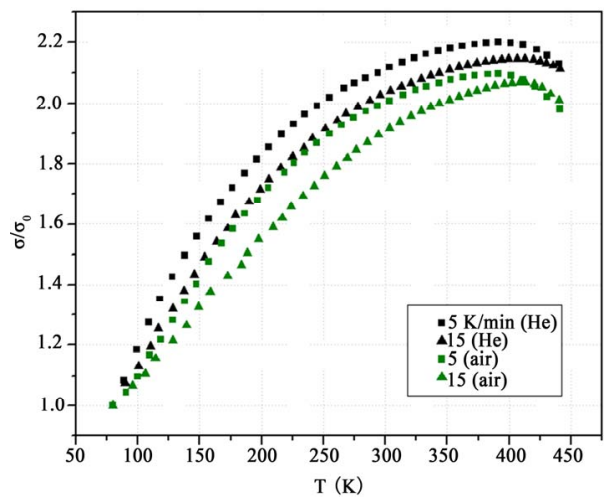

Figure 1. The reduced conductivity $\sigma / \sigma_{0}$ of a $120 \mathrm{~nm}$ thick PEDOT: PSS film, where $\sigma_{0}$ is the conductivity at $T=80 \mathrm{~K}$, as a function of $T$, for two heating rates 5 and $15 \mathrm{~K} / \mathrm{min}$, under $\mathrm{He}$ and atmospheric air. 
From Figure 1 it can be seen that for temperatures higher than $T_{m}$ the decline of the electrical conductivity with increasing temperature $(\mathrm{d} \sigma / \mathrm{dT}<0)$ is slower in the samples heated under $\mathrm{He}$, indicating the negative role of the oxygen and the moisture on the electrical conductivity. Any connection between $\mathrm{T}_{\mathrm{m}}$ and a possible glass transition at this temperature must be dismissed. Indeed, from the literature, DSC results for PEDOT:PSS show that this material does not have a specific $T_{g}$ due to the strong ionic bonding between PEDOT and PSS [7,25].

In Figure 1 the experimental points near the lowest temperature $(80 \mathrm{~K})$ coincide. This can be attributed to the fact that at this low temperature, oxygen and moisture have not yet affected the polymer chains and the rearrangement of the polymer chains is restricted. Moreover, another coincidence is observed at the highest temperature $(\sim 445 \mathrm{~K})$. Here, it is different for the two surrounding atmospheres. This might be due to the fact that at this high temperature the degree of the crystallinity has attained its maximum value and the heating rate plays only a negligible role to the phenomenon.

\subsection{Isotherms of the Electrical Conductivity of PEDOT:PSS under Atmospheric Air vs Time}

To investigate the influence of the antagonistic processes on the electrical conductivity, the change of $\sigma / \sigma_{\mathrm{i}}$, where $\sigma_{\mathrm{i}}$ is the initial conductivity, was measured on seven PEDOT:PSS films $120 \mathrm{~nm}$ thick. Each sample was heated under atmospheric air for $1 \mathrm{~h}$ at one of the following temperatures $373,393,423,438,443,448$ and $463 \mathrm{~K}$ and the results are shown in Figure 2 for the first six samples and in the inset for the last one. The heating rate from room temperature to each of the above mentioned temperature was $10 \mathrm{~K} / \mathrm{min}$.

For $\mathrm{T}=373$ and $393 \mathrm{~K}$ a systematic reduction of the conductivity with the heating time $\mathrm{t}$ is observed, more intense for the higher temperature. This can be explained taking into account that from the different mechanisms which determine the electrical conductivity, the irreversible changes of the polymer backbone in the presence of the oxygen and moisture of the air prevail.

However, at $423 \mathrm{~K}$ the reduction of the conductivity is less than at $393 \mathrm{~K}$ indicating that the improvement of the crystallinity, which counteracts the reduction of the conductivity, starts to affect the phenomenon $[15,26]$. The existence of this mechanism becomes more obvious for higher heating temperatures 438, 443 and $448 \mathrm{~K}$. The conductivity increases for the first 7 to $12 \mathrm{~min}$ and then decays. From Figure 2, it can be deduced that the increase of the crystallinity contribution to the conductivity takes its maximum at about $443 \mathrm{~K}$ after heating of about $10 \mathrm{~min}$. This is in accordance with J. Huang et al. (2003) results, reported that the conductivity of PEDOT:PSS films exhibits a maximum after 10 - 15 min of heat treatment in $\mathrm{N}_{2}$. The conductivity in the latter case was measured following equilibration at room temperature.

In the inset of Figure 2 the behavior of the seventh sample is shown, which was heated at $463 \mathrm{~K}$. The thermal treatment at this temperature for $1 \mathrm{~h}$ leads to an abrupt decrease of the conductivity to about half of its initial value. The thermal degradation is so intense that any possible improvement of the crystallinity is completely overwhelmed.

\subsection{Influence of the Surrounding Atmosphere on the Electrical Conductivity of PEDOT:PSS}

In order to illustrate the difference between the antagonistic processes under the two investigated environments and estimate the time interval during which the mechanism enhancing the conductivity prevails, measurements on the electrical conductivity as a function of heat treatment time at constant temperature were performed, as shown in Figure 3.

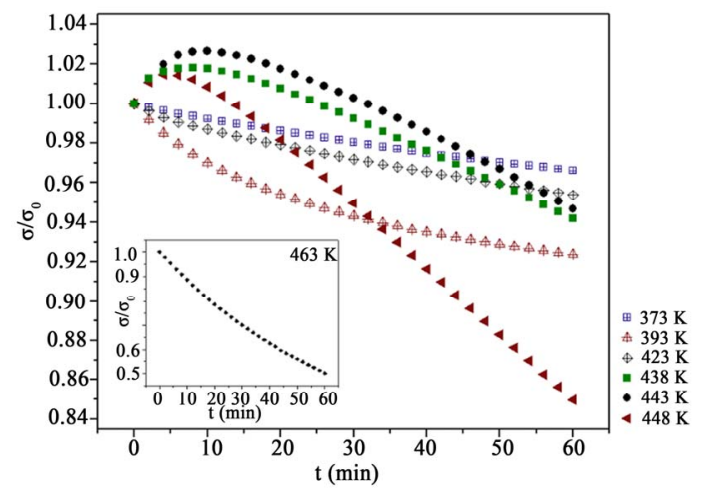

Figure 2. The reduced conductivity $\sigma / \sigma_{0}$, where $\sigma_{0}$ is the conductivity at $t=0 \mathrm{~min}$, for seven $120 \mathrm{~mm}$ thick PEDOT: PSS films under atmospheric air for an $1 \mathrm{~h}$ heating at the seven temperatures shown. The inset concerns the sample heated at $463 \mathrm{~K}$.

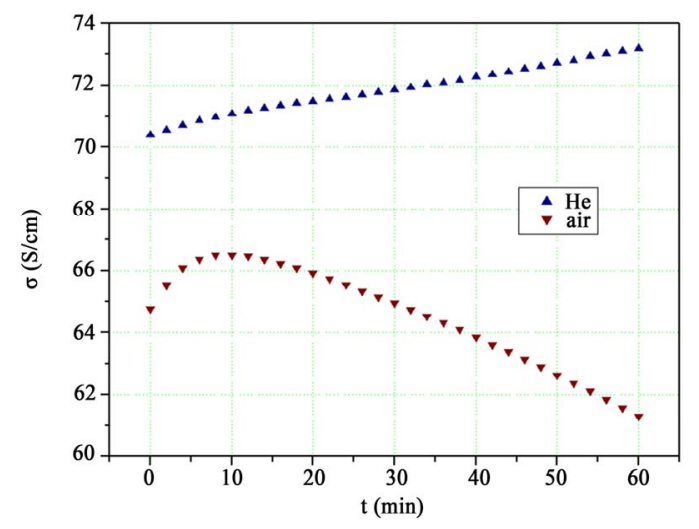

Figure 3. The change of the conductivity of two PEDOT: PSS $120 \mathrm{~nm}$ thick films, as a function of heating time $t$ at $443 \mathrm{~K}$ under inert He and atmospheric air. 
The first sample was heated under inert He at $443 \mathrm{~K}$, the characteristic temperature, as deduced from Figure 2, in which the maximum increase of $\sigma / \sigma_{0}$ was observed under the thermal treatment. The conductivity increased continuously with a slightly higher rate at the first $10 \mathrm{~min}$ due to the crystallization improvement. The behavior of the other sample heated under atmospheric air is quite different, as has been already discussed, for the first $10 \mathrm{~min}$ its conductivity increases and then steadily decreases.

From these two curves of Figure 3 the decisive role of the surrounding atmosphere becomes apparent. Under inert atmosphere the deterioration of the polymer structure seems to have only minor influence on the conductivity. On the contrary, the presence of oxygen and moisture promotes the irreversible structural modifications of the PEDOT:PSS chains, strongly reducing the conductivity.

\section{Summary and Conclusions}

The competition between the increase of the electrical conductivity $\sigma$ due to the activation of charge carriers and the improvement of the crystallinity in PEDOT:PSS films with heating and the simultaneous decrease of $\sigma$ caused by irreversible structural degradation of the polymer chains were investigated, under inert $\mathrm{He}$ and in atmospheric air.

The experimental results showed that the increase of $\sigma$ due to crystallization gets apparent for heating the polymer at $423 \mathrm{~K}$ and maximizes at $443 \mathrm{~K}$ for heating time about $10 \mathrm{~min}$. Moreover, the heating rate $5 \mathrm{~K} / \mathrm{min}$ results in a higher conductivity than the quicker selected rate of $15 \mathrm{~K} / \mathrm{min}$, since the slow heating rate allows the crystallization to develop more efficiently. The electrical conductivity $\sigma$ increases with $\mathrm{T}$ up to a certain temperature $\mathrm{T}_{\mathrm{m}}$ and then decreases. This indicates that for $\mathrm{T}<\mathrm{T}_{\mathrm{m}}$ the heat activation of carriers and the improvement of the crystallinity dominate, though for $\mathrm{T}>\mathrm{T}_{\mathrm{m}}$ the degradation of the chains and the deterioration of the crystallinity play the first role. A remarkable characteristic is that $T_{m}$ is independent from He or ambient atmosphere, depending exclusively on the heating rate. This indicates that the chain degradation due to the oxygen and the moisture of the air does not affect the temperature at which equilibrium is established between the two competitive processes.

Finally, the destructive role of oxygen and moisture of the atmospheric air on PEDOT: PSS becomes apparent from the experimental results and this parameter should be taken into account for understanding the lifetime performance of organic optoelectronic devices containing PEDOT:PSS buffer layers.

\section{Acknowledgements}

This work was partially financed by the Research Committee of the University of Patras under the framework of the "K. Karatheodori" program for basic research strengthening. S. A. Choulis would like to thank Cyprus Research Promotion foundation for funding the development of the Molecular Electronics and Photonics Research Unit under research grant "NEA YПО $\Delta \mathrm{OMH}$

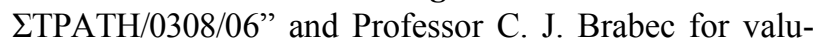
able discussions.

\section{REFERENCES}

[1] A. Elschner, F. Bruder, H.-W. Heuer, F. Jonas, A. Karbach, S. Kirchmeyer, S. Thurm and R. Wehrmann, "PEDT/PSS for Efficient Hole-Injection in Hybrid Organic Light-Emitting Diodes," Synthetic Metals, Vol. 111-112, 2000, pp. 139-143. doi:10.1016/S0379-6779(99)00328-8

[2] R. Pacios and D. D. C. Bradley, "Charge Separation in Polyflourene Composites with Internal Donor/acceptor Heterojunctions," Synthetic Metals, Vol. 127, No. 1-3, 2002, pp. 261-265. doi:10.1016/S0379-6779(01)00629-4

[3] V. H. Schmidt, L. Lediaev, J. Polasik and J. Hallenberg, "Piezoelectric Actuators Employing PVDF Coated with Flexible PEDOT-PSS Polymer Electrodes," IEEE Transactions on Dielectrics and Electrical Insulation, Vol. 13, No. 5, 2006, pp. 1140-1148. doi: $10.1109 /$ tdei.2006.247842

[4] S. K. M. Jönsson, W. R. Salaneck and M. Fahlman, "Spectroscopy of Ethylenedioxythiophene-Derived Systems: From Gas Phase to Surfaces and Interfaces Found in Organic Electronics," Journal of Electron Spectroscopy and Related Phenomena, Vol. 137-140, 2004, pp. 805-809. doi:10.1016/j.elspec.2004.02.120

[5] S. A. Choulis, V.-E. Choong, A. Patwardhan, M. K. Mathai and F. So, "Interface Modification to Improve Hole-Injection Properties in Organic Electronic Devices," Advanced Functional Materials, Vol. 16, No. 8, 2006, pp. 1075-1080. doi:10.1002/adfm.200500443

[6] J. Chung, K. H. Kim, J. C. Lee, M. K. Kim and H. J. Shin, "Spectroscopic Investigation of Polymer Light-Emitting Device Degradation," Organic Electronics, Vol. 9, No. 5, 2008, pp. 869-872. doi:10.1016/j.orgel.2008.06.009

[7] K. Fehse, R. Meerheim, K. Walzer, K. Leo, W. Lövenich and A. Elschner, "Lifetime of Organic Light Emitting Diodes on Polymer Anodes," Applied Physics Letters, Vol. 93, No. 8, 2008, pp. 083303-1-083303-3. doi:10.1063/1.2975369

[8] S. J. Martin, R. A. L. Jones, M. Geoghegan, A. M. Higgins, I. Grizzi, J. J. M. Halls, S. Kirchmeyer and R. M. Dalgliesh, "Current-Induced Chain Migration in Semiconductor Polymer Blends," Physical Review B, Vol. 71, No. 8, 2005, pp. 081308-1-081308-4. doi:10.1103/PhysRevB.71.081308

[9] C. Gärditz, A. Winnacker, F. Schindler and R. Paetzold, "Impact of Joule Heating on the Brightness Homogeneity of Organic Light Emitting Devices," Applied Physics Letters, Vol. 90, No. 10, 2007, pp. 103506-1-103506-3. doi:10.1063/1.2711708

[10] C.-H. Chang and S.-A. Chen, "Effect of Ionization Poten- 
tial Change in Poly(3,4-ethylenedioxythiophene): Poly (Styrenesulfonic Acid) on the Performance of Polymer Light Emitting Diodes Due to Its Reaction with Indium Tin Oxide," Applied Physics Letters, Vol. 91, No. 10, 2007, pp. 103514-1-103514-3. doi:10.1063/1.2759474

[11] P. Tehrani, A. Kanciurzewska, X. Crispin, N. D. Robinson, M. Fahlman and M. Berggren, "The Effect of pH on the Electrochemical Over-Oxidation in PEDOT:PSS Films," Solid State Ionics, Vol. 177, No. 39-40, 2007, pp. 3521-3527. doi:10.1016/j.ssi.2006.10.008

[12] E. Vitoratos, S. Sakkopoulos, E. Dalas, N. Paliatsas, D. Karageorgopoulos, F. Petraki, S. Kennou and S. A. Choulis, "Thermal Degradation Mechanisms of PEDOT:PSS," Organic Electronics, Vol. 10, No. 1, 2009, pp. 61-66. doi:10.1016/j.orgel.2008.10.008

[13] J. Huang, P. F. Miller, J. C. de Mello, A. J. de Mello and D. D. C. Bradley, "Influence of Thermal Treatment on the Conductivity and Morphology of PEDOT/PSS Films," Synthetic Metals, Vol. 139, No. 3, 2003, pp. 569-572. doi:10.1016/S0379-6779(03)00280-7

[14] R. Kiebooms, A. Aleshin, K. Hutchison, F. Wudl and A. Heeger, "Doped Poly(3,4-ethylenedioxythiophene) Films: Thermal, Electromagnetical and Morphological Analysis," Synthetic Metals, Vol. 101, No. 1-3, 1999, pp. 436-437. doi:10.1016/S0379-6779(98)01121-7

[15] K. E. Aasmundtveit, E. J. Samuelsen, L. A. A. Pettersson, O. Inganäs, T. Johansson and R. Feidenhans, "Structure of Thin Films of Poly(3,4-ethylenedioxythiophene)," Synthetic Metals, Vol. 101, No. 1-3, 1999, pp. 561-564. doi:10.1016/S0379-6779(98)00315-4

[16] H. H. Wieder, "Laboratory Notes of Electrical and Galvanomagnetic Measurements,” Elsevier, Amsterdam, 1979.

[17] U. Lang, P. Rust, B. Schoberle and J. Dual, "Piezoresistive Properties of PEDOT:PSS," Microelectronic Engineering, Vol. 86, No. 3, 2009, pp. 330-334 doi:10.1016/j.mee.2008.10.024

[18] A. M. Nardes, M. Kemerink, M. M. de Kok, E. Vinken, K. Maturova and R. A. J. Janssen, "Conductivity, Work Function, and Environmental Stability of PEDOT:PSS Thin Films treated with Sorbitol," Organic Electronics, Vol. 9, No. 5, 2008, pp. 727-734. doi:10.1016/j.orgel.2008.05.006

[19] J. Huang, P. F. Miller, J. S. Wilson, A. J. de Mello, J. C. de Mello and D. D. C. Bradley, "Investigation of the Effects of Doping and Post-Deposition Treatments on the Conductivity, Morphology, and Work Function of Poly(3,4-ethylenedioxythiophene)/Poly(Styrene Sulfonate) Films," Advanced Functional Materials, Vol. 15, No. 2, 2005, pp. 290-296. doi:10.1002/adfm.200400073

[20] M. M. de Kok, M. Buechel, S. I. E. Vulto, P. van de Weijer, E. A. Meulenkamp, S. H. P. M. de Winter, A. J. G. Mank, H.J.M. Vorstenbosch, C.H.L. Weijtens and V. van Elsbergen, "Modification of PEDOT:PSS as Hole Injection Layer in Polymer LEDs," Physica Status Solidi (a), Vol. 201, No. 6, 2004, pp. 1342-1359. doi: $10.1002 /$ pssa.200404338

[21] R. R. Smith, A. P. Smith, J. T. Stricker, B. F. Taylor and M. F. Durstock, "Layer-by-Layer Assembly of Poly(3,4ethylenedioxythiophene):Poly(styrenesulfonate)," Macromolecules, Vol. 39, No. 18, 2006, pp. 6071-6074. doi: $10.1021 / \mathrm{ma} 060775 \mathrm{~d}$

[22] C. S. S. Sangeeth, M. Jaiswal and R. Menon, "Correlation of Morphology and Charge Transport in Poly(3,4-ethylenedioxythiophene)-Polystyrenesulfonic Acid (PEDOT:PSS) Films," Journal of Physics: Condensed Matter, Vol. 21, No. 7, 2009, Article ID: 072101. doi:10.1088/0953-8984/21/7/072101

[23] A. B. Kaiser, "Electronic Transport Properties of Conducting Polymers and Carbon Nanotubes," Reports on Progress in Physics, Vol. 64, No. 1, 2001 pp. 1-49. doi:10.1088/0034-4885/64/1/201

[24] B. Dufour, P. Rannou, D. Djurado, M. Zagorska, I. Kulszewicz-Bajer and A. Pron, "The Role of Chain and Dopant Engineering in the Preparation of Processible Conducting Polymers with Desired Properties," Synthetic Metals, Vol.135/136, 2003, pp. 63-68. doi:10.1016/S0379-6779(02)00538-6

[25] Y. Yoshioka and G. E. Jabbour, "Inkjet Printing and Patterning of PEDOT-PSS: Application to Optoelectronic Devices," In: T. Skotheim and J. Reynolds, Eds., Conjugated Polymers, CRC Press, Taylor and Francis Group, New York, 2007.

[26] J. Li, L. Fan and C. P. Wong, "Influence of Thermal Treatment on the Conductivity of PEDT Films," Proceedings IEEE 9th International Symposium of Advanced Packaging Materials, Atlanda, 24-26 March 2004, pp. 204-207. 